\title{
Stability of Nb-Ti Rutherford Cables Exhibiting Different Contact Resistances
}

\author{
G. P. Willering, A. P. Verweij, J. Kaugerts, and H. H. J. ten Kate
}

\begin{abstract}
Dipole magnets for the so-called SIS-300 heavy-ion synchrotron at GSI are designed to generate $6 \mathrm{~T}$ with a field sweep rate of $1 \mathrm{~T} / \mathrm{s}$. It is foreseen to wind the magnets with a 36 strands $\mathrm{Nb}$-Ti Rutherford cable. An important issue in the cable design is sufficiently low AC loss and stability as well. In order to keep the AC loss at low level, the contact resistance between crossing strands $R_{c}$ is kept high by putting a stainless steel core in the cable. The contact resistance between adjacent strands $R_{a}$ is controlled by oxidation of the Sn-Ag coating of the strands, like in the LHC. In order to investigate the effect of $R_{a}$ on the stability of the cable, we prepared four samples with different $R_{a}$ by varying the heat treatment and applying a soldering technique, resulting in values between $1 \mu \Omega$ to $9 \mathrm{~m} \Omega$. The stability of each sample against transient point-like heat pulses was measured. The results of the stability experiments and a comparison with calculations using the network model CUDI are presented. It is concluded that variation of $\mathbf{R}_{\mathbf{a}}$ has a strong influence on cable stability and that optimization of $R_{a}$ is mandatory to properly design the cable for the SIS-300 magnets, or likewise for similar magnets that might be used at CERN for a possible LHC injector upgrade.
\end{abstract}

Index Terms-Magnets, minimum quench energy, stability, superconducting cables.

\section{INTRODUCTION}

$\mathbf{T}$ HE stability of a superconducting cable against local disturbances is in general described by a curve presenting the Quench Energy QE as a function of the normalized current $I / I_{\mathrm{c}}$ as shown in Fig. 1 [1], where $I_{\mathrm{c}}$ is the critical current. Normally this curve exhibits a sharp 'kink' separating two distinctive stability regimes. The current at which the kink occurs is called $I_{\text {kink }}$. Regime $\mathrm{I}$ is the high $I / I_{\mathrm{c}}$ regime, where cable stability behavior is similar to the behavior of a single strand. Regime II is the low $I / I_{\mathrm{c}}$ regime, where current redistribution increases stability.

In order to produce cables that are stable at operating point against short point like disturbances it is preferable to stay in stability regime II, where stability increases by more than one order of magnitude. In measurement and simulation we will demonstrate how heat treatment or partial soldering can considerably influence cable stability in this regime.

This study was performed to be able to choose the correct inter-strand contact of the cables for the FAIR SIS 300 high ramp rate magnets [2]. GSI prepared cored Rutherford cables

Manuscript received August 28, 2007

G. P. Willering and H. H. J. ten Kate are with the CERN, Geneva, Switzerland, and also with the University of Twente, Enschede, The Netherlands (e-mail: gerard.willering@cern.ch).

A. P. Verweij is with CERN, Geneva, Switzerland.

J. Kaugerts is with GSI, Darmstadt, Germany.

Digital Object Identifier 10.1109/TASC.2008.920560

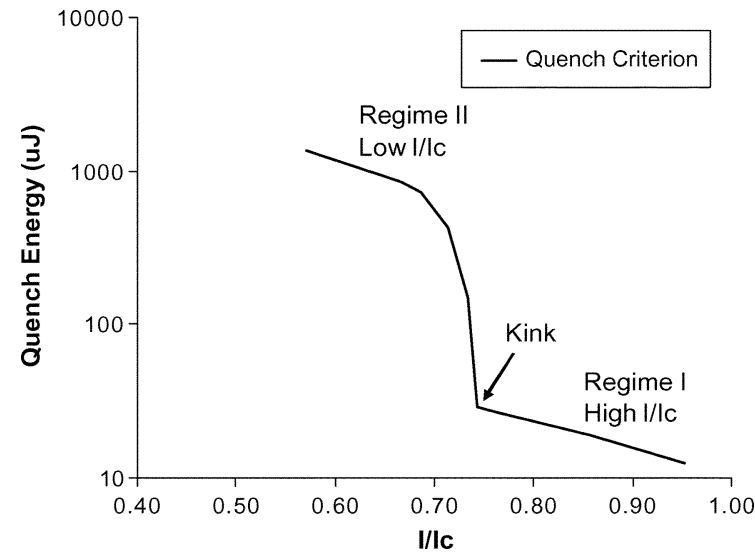

Fig. 1. Quench Energy versus $I / I_{\mathrm{c}}$ curve with characteristic points and area's, showing regime I on the right side of the kink and regime II on the left side.

with four different surface preparations in order to vary the contact resistance between adjacent strands $\left(R_{\mathrm{a}}\right)$ as much as possible. Note that GSI requires a high $R_{\mathrm{a}}$-value in order to keep the $\mathrm{AC}$ loss at a reasonable level. The stability of the four samples is measured at CERN in the cable test facility FRESCA [3]. Simulations are performed with the network model CUDI [4], and comparison to the experimental curves, gave us a profound insight in the mechanisms of cable stability and the effect of the strand surface treatment on the stability.

In Table I the main design parameters of the tested strand and cable are listed. The cable is very similar to the outer layer cable of the LHC dipole magnets at CERN, with the exception of a smaller filament diameter, a smaller filament twist, and the presence of a core. Four identical cable pieces were given different treatments. The four samples are denoted "low- $R_{\mathrm{a}}$ ", "medium- $R_{\mathrm{a}}$ ", "high- $R_{\mathrm{a}}$ ", and "ultrahigh- $R_{\mathrm{a}}$ " and some additional measured characteristics are shown in Table II. $R_{\mathrm{a}}$ is measured according to [5]. These surface treatments did not only change the $R_{\mathrm{a}}$-value, but at the same time also the interstrand heat conductivity $k_{\text {is }}$, the RRR value, and may possibly affect the heat transfer between the strand surface and the helium $\left(k_{\mathrm{He}}\right)$. Note that when these cables would be used in a magnet, the final curing of the coil would again change the values of these parameters.

\section{Factors Influencing CABle Stability}

Based on our experiments and modeling, the criteria for developing a quench or recovery in a cable can be described according to Table III [6].

In regime I the stability behavior is similar to that of a single strand. In this regime current redistribution does not affect the quench level and the value of $R_{\mathrm{a}}$ does therefore play no role. The most significant parametric effect on stability is caused by a change in $k_{\mathrm{He}}$, followed by $k_{\text {is }}$ and the RRR-value. 
TABLE I

DESIGN PARAMETERS OF STRAND AND CABLE

\begin{tabular}{lc}
\hline \hline & Strand \\
\hline Strand diameter & $0.825 \mathrm{~mm}$ \\
Filament diameter & $6.0 \mu \mathrm{m}$ \\
Filament twist pitch & $15 \mathrm{~mm}$ \\
Cu/Nb-Ti ratio & 1.95 \\
Strand coating & $\mathrm{Sn}-\mathrm{Ag}($ Staybrite $)$ \\
Critical current density $J_{\mathrm{c}}$ & $2700 \mathrm{~A} / \mathrm{mm}^{2}(5 \mathrm{~T}, 4.2 \mathrm{~K})$ \\
Critical current density $J_{\mathrm{c}}$ & $2130 \mathrm{~A} / \mathrm{mm}^{2}(6 \mathrm{~T}, 4.2 \mathrm{~K})$ \\
\hline \multicolumn{2}{c}{ Rutherford Cable } \\
\hline Strand number & 36 \\
Transposition pitch & $100 \mathrm{~mm}$ \\
Width & $15.1 \mathrm{~mm}$ \\
Thickness, thin edge & $1.362 \mathrm{~mm}$ \\
Thickness, thick edge & $1.598 \mathrm{~mm}$ \\
Mid-thickness & $1.480 \mathrm{~mm}$ \\
Core size and material & $13 \mathrm{~mm}$ wide, $25 \mu \mathrm{m}$ thick, \\
& Stainless steel 304 \\
Crossover resistance $R_{\mathrm{c}}$ & $20 \mathrm{~m} \Omega$ \\
\hline \hline
\end{tabular}

TABLE II

MEASURED CABLE PARAMETERS

\begin{tabular}{ccccc}
\hline \hline Identification & Treatment & RRR & $R_{\mathrm{a}}(\mu \Omega)$ & $I_{\mathrm{c}}(\mathrm{A}) 6 \mathrm{~T}, 4.3 \mathrm{~K}$ \\
\hline low- $\mathrm{R}_{\mathrm{a}}$ & $\begin{array}{c}\text { partially soldered at } 200 \\
\text { o }\end{array}$ & 346 & $<1$ & 14689 \\
$\begin{array}{c}{ }^{\circ} \mathrm{C}+3 \text { minutes at } 250{ }^{\circ} \mathrm{C} \\
\text { medium- }\end{array}$ & & & \\
high- $\mathrm{R}_{\mathrm{a}}$ & $\begin{array}{c}\text { no treatment } \\
\text { heat treatment }\end{array}$ & 89 & $60-70$ & 14240 \\
ultrahigh- $\mathrm{R}_{\mathrm{a}}$ & $\begin{array}{c}18 \mathrm{~h} \text { at } 215^{\circ} \mathrm{C} \text { in vacuum } \\
+58 \mathrm{~h} \text { at } 210^{\circ} \mathrm{C} \text { in air }\end{array}$ & 272 & $800-700$ & 14741 \\
& & & 14112 \\
\hline \hline
\end{tabular}

TABLE III

QUENCH CRITERIA FOR THE TWO MAIN STABILITY REGIMES

\begin{tabular}{lll}
\hline \hline \multirow{2}{*}{ Regime I } & No Quench & No normal zone occurs in the heated strand. \\
\cline { 2 - 3 } Quench & $\begin{array}{l}\text { Normal zone occurs in the heated strand, no } \\
\text { recovery possible. }\end{array}$ \\
\hline No Quench & $\begin{array}{l}\text { A normal zone in the heated strand leads to current } \\
\text { redistribution, but the other strands stay } \\
\text { superconductive. Recovery takes place. }\end{array}$ \\
\cline { 2 - 3 } Regime II & Quench & $\begin{array}{l}\text { Normal zone occurs in the heated strand, causing } \\
\text { current redistribution leading to a normal zone in } \\
\text { the adjacent strand. No recovery possible. }\end{array}$ \\
\hline \hline
\end{tabular}

In regime II, however, the stability is also influenced by $R_{\mathrm{a}}$, because current redistribution takes place as soon as a normal zone is created in one strand. In order to improve the stability a shift of the kink to higher $I / I_{\mathrm{c}}$ and an increase of the quench level left from the kink are highly desirable. This can be achieved by increasing $k_{\mathrm{He}}$ and the Residual Resistance Ratio of the copper RRR, and decreasing $k_{\text {is }}$ and $R_{\mathrm{a}}$. Note that measurements and simulations show that current redistribution mainly occurs through the adjacent contacts and not through the crossing contacts. This is certainly true for $R_{\mathrm{c}} \gg R_{\mathrm{a}}$, but it even holds when these parameters have the same order of magnitude.

\section{EXPERIMENTAL SETUP}

The stability measurements were performed in the CERN cable test facility FRESCA. For these tests we use cable samples of $2.4 \mathrm{~m}$ length of which $1 \mathrm{~m}$ was positioned in the external magnetic field which was applied parallel to the broad face of the cable. On the broad face a $50 \mu \mathrm{m}$ thick adhesive Kapton

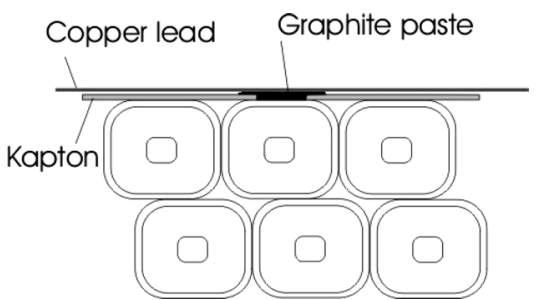

Fig. 2. Mounting a the graphite paste heater on a strand surface.

tape was placed with pre-fabricated holes of $0.5 \mathrm{~mm}$ diameter. These holes are positioned above single strands and covered with a small amount of graphite epoxy paste (Eccobond $60 \mathrm{~L}$ ). A copper strip of $1 \mathrm{~mm}$ wide was placed over the paste and held in place by adhesive Kapton tape. More details can be found in [7]. During the curing of the epoxy, pressure was applied in such a way that a final resistance between 1 and $2 \Omega$ is reached. A similar set-up of heaters was used by [8]-[10].

In Fig. 2 the mounting of the heaters is shown schematically. Usually three heaters per strand are positioned on the surface of about 5 consecutive strands. In this way the heaters can act as voltage taps as well, by measuring the voltages across single strands. Here we limit ourselves to the stability behavior of the central heaters.

Heat is deposited in the strand by applying a square electrical pulse with a duration of $100 \mu \mathrm{s}$ and powers up to $40 \mathrm{~W}$. The superconducting strand is used as a return current lead. Only the heat entering the strand before the quench decision moment (QDM) takes place has to be taken into account. Although the heaters are in direct contact with the strand, the heat pulse will enter the strand with a delay. Modeling is performed [11] showing the exact shape of the heat pulse entering the strand. By integrating the heat from the start of the pulse to the QDM, one obtains the effective heat that has to be taken into account. The scaling factor $F_{\mathrm{sc}}$ is defined as the ratio between the effective quench energy $(\mathrm{QE})$ and the input pulse energy $\left(E_{\text {inp }}\right)$. As justified in [11] we will apply $F_{\mathrm{sc}}=0.4$ in regime I (having a QDM of about $100 \mu \mathrm{s}$ ) and $F_{\mathrm{sc}}=0.9$ in regime II (having a QDM of about $0.5 \mathrm{~ms}$ ).

\section{Measurement Results}

The QE measurement is performed by ramping the current to the desired level with constant temperature and field. A heat pulse is deposited onto one strand of the cable. If no quench follows, the cable is quenched manually to prevent non-uniform current distribution due to the creation of local normal zones. Consecutive pulses are applied to determine the quench energy within $5 \%$ accuracy. The scaling factor $F_{\mathrm{sc}}$ is applied in order to obtain the effective quench energy $Q E$. Such experiments are performed for several heaters per sample and at different temperatures and fields. For clarity, here only data obtained at $4.3 \mathrm{~K}$ and $6 \mathrm{~T}$ are presented.

Fig. 3 shows the obtained QE from the stability measurements for the four cable samples. The position of the kink varies quite dramatically from $I / I_{\mathrm{c}} \approx 0.62$ for the highest $R_{\mathrm{a}}$ to $I / I_{\mathrm{c}}>1$ for the lowest $R_{\mathrm{a}}$ of the cable. It is clearly visible that for three curves Regime I is not significantly affected by the change of parameters. This is expected, because in as regime $k_{\text {is }}$ plays a minor role to cool the strand as compared to the helium cooling. The $R_{\mathrm{a}}$-value itself is also unimportant because current redistribution as a stabilizing feature does not occur. For the low- $R_{\mathrm{a}}$ sample current redistribution always takes place in 


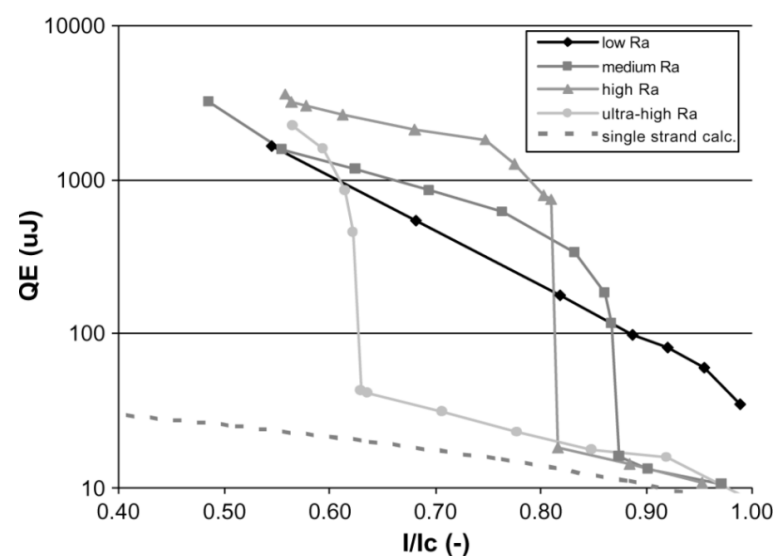

Fig. 3. Quench energy plotted against $I / I_{\mathrm{c}}$ for the four differently prepared cable samples at a temperature of $4.3 \mathrm{~K}$ and an applied field of $6 \mathrm{~T}$. The dashed line shows the calculated QE for a comparable single strand with a $100 \mu$ s square pulse.

TABLE IV

CONTACT SURfaces AND HeLIUM Volume

\begin{tabular}{ll}
\hline \hline Contact surface between strand and Helium $A_{\mathrm{He}}\left(\mathrm{mm}^{2}\right)$ & 643 \\
Contact surface between adjacent strands $A_{\mathrm{a}}\left(\mathrm{mm}^{2}\right)$ & 144 \\
Helium volume per strand $V_{\mathrm{He}}\left(\mathrm{mm}^{3}\right)$ & 12.3 \\
\hline \hline
\end{tabular}

multiple strands and stability regimes cannot be defined. The quench level left of the kink tend to increase if the kink moves to lower $I / I_{\mathrm{c}}$ values. This can be attributed to the lower thermal interstrand conductivity.

\section{Simulation Results}

Simulations were performed with the network model CUDI to better understand the measurements. In CUDI, the cable is discretized in small strand sections and all currents and temperatures are solved as functions of time. The main equation that needs to be solved in the thermal module for every small time step dt is:

$$
\begin{array}{r}
c_{p} \frac{\Delta T_{s}}{d t} V_{s}=P_{i n p}+I_{s}^{2} R_{s}+I_{a}^{2} R_{a}-k_{H e}\left(T_{s}-T_{H e}\right) A_{H e} \\
-k_{i s}\left(T_{s}-T_{a}\right) A_{a}-k_{s} \frac{d T_{s}}{d x} A_{s}
\end{array}
$$

With $c_{\mathrm{p}}$ specific heat, $T_{\mathrm{s}}$ temperature, $V_{\mathrm{s}}$ volume, $I_{\mathrm{s}}$ the current, $R_{\mathrm{S}}$ resistance, $k_{\mathrm{S}}$ thermal conductivity and $A_{\mathrm{S}}$ the cross-section of a given strand section. $P_{\text {inp }}$ is the power from the external pulse, $I_{\mathrm{a}}$ the current through the adjacent contacts, $T_{\mathrm{He}}$ the helium temperature, $T_{\mathrm{a}}$ the temperature of the adjacent strand, $A_{\mathrm{a}}$ the contact surface between adjacent strands and $d x$ the spatial step size in longitudinal direction.

For this model we need an accurate description of strand and cable parameters, as described in Tables I and II. In [12] the topology of the LHC type II cable is described in detail, revealing the surfaces and volumes of the strands and helium. For our cable we assume that due to the creep of the kapton tape no helium exists on the outer surface of the cable, but only on the inner surface. The keystone angle is ignored and the surfaces, volumes and contact resistances are kept constant across the cable width. Table IV shows the given parameters per meter strand averaged over the cable width as used in the simulations.

Heat transfer to Helium I consists of multiple regimes [13]. Simulations show that the most important regime is the transient cooling regime. When the accumulated heat transferred

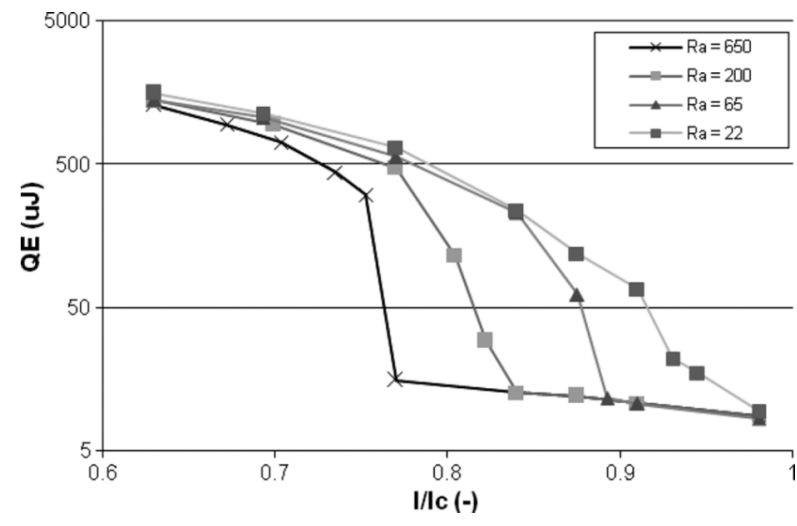

Fig. 4. Quench Energy versus $I / I_{c}$ showing the effect of varying $R_{\mathrm{a}}$ between 22 and $650 \Omega$, with $\mathrm{RRR}=89$.

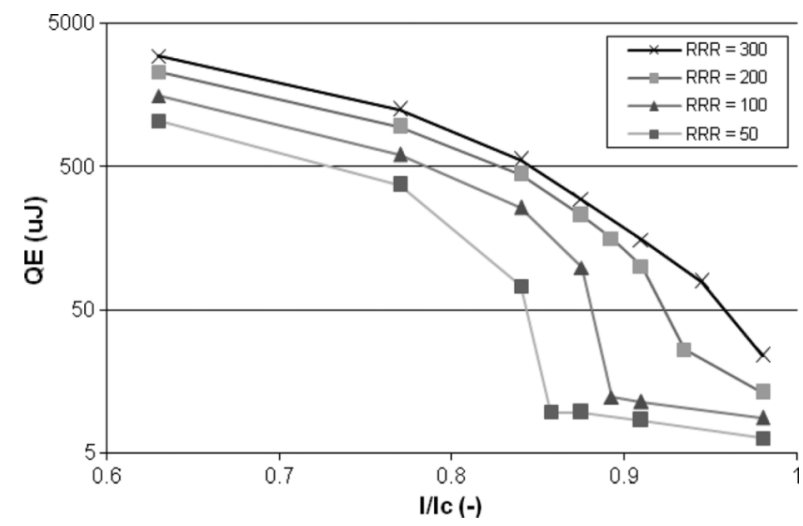

Fig. 5. Quench Energy versus $I / I_{c}$ showing the effect of varying RRR between 50 and 300 , with $R_{\mathrm{a}}=65$.

into the helium exceeds a certain limit, the nucleate boiling regime starts. Because this limit is governed primarily by the physical properties of helium and to a much smaller extent by the strand surface conditions a value of $20 \mathrm{~J} / \mathrm{m}^{2}[14]$ is assumed here. The unknown parameters available to fit the simulated to the measured curves are:

- Interstrand heat conductivity parameter $f_{\text {is }}\left[\mathrm{W} / \mathrm{m}^{2} /\right.$ $\left.\mathrm{K}^{n-i n t}\right]$ used in:

$$
k_{i s}=f_{i s} \cdot T^{n_{i s}}
$$

- Transient helium cooling parameter $\alpha_{\text {trans }}\left[\mathrm{W} / \mathrm{m}^{2} /\right.$ $\left.\mathrm{K}^{\mathrm{n} \_ \text {trans }}\right]$ used in:

$$
k_{H e}=a_{\text {trans }} \cdot\left(T_{s}^{n_{\text {trans }}}-T_{\text {bath }}^{n_{\text {trans }}}\right)
$$

with $n_{\text {is }}=2.25$ [15] and with $n_{\text {trans }}=4$ [13]

Figs. 4 and 5 show results of simulations with varying $R_{\mathrm{a}}$ and RRR.

To better understand the influence of $f_{\text {is }}$ and $\alpha_{\text {trans }}$ on the QE first simulations by varying these parameters independently as shown in the Figs. 6 and 7 are performed. Note that the effect of changing a combination of parameters can make the effects stronger or weaker. The main observation is that an increase in $f_{\text {is }}$ decreases the quench level in Regime II and an increase in $\alpha_{\text {trans }}$ shifts the position of the kink to the right and increases the quench level in Regimes I and II.

The simulated curves that fit the measured curves best are shown in Fig. 8. Table $\mathrm{V}$ shows the parameters used for these best fits.

The expected decrease in $f_{\text {is }}$ (and hence $k_{\text {is }}$ ) for increasing $R_{\mathrm{a}}$ is clear. From literature it is well known that the Kapitza conduc- 


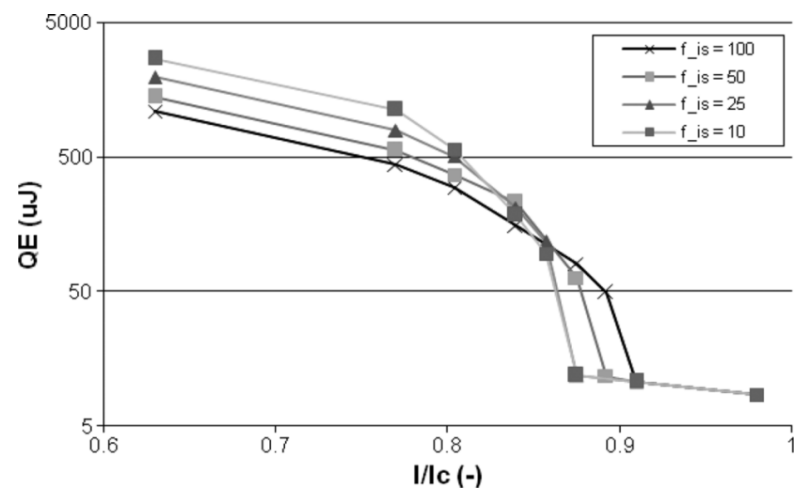

Fig. 6. Quench Energy versus $I / I_{c}$ showing the effect of varying $f_{\text {is }}$ between 10 and 100 .

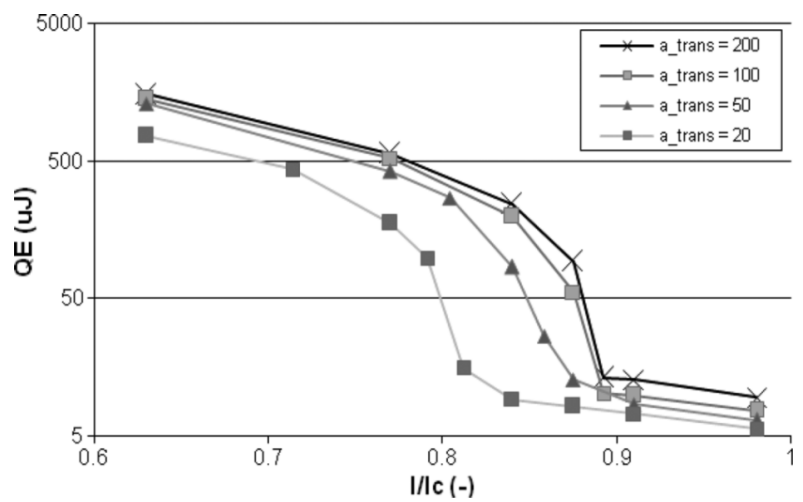

Fig. 7. Quench Energy versus $I / I_{c}$ showing the effect of varying $\alpha_{\text {trans }}$ between 20 and 200 .

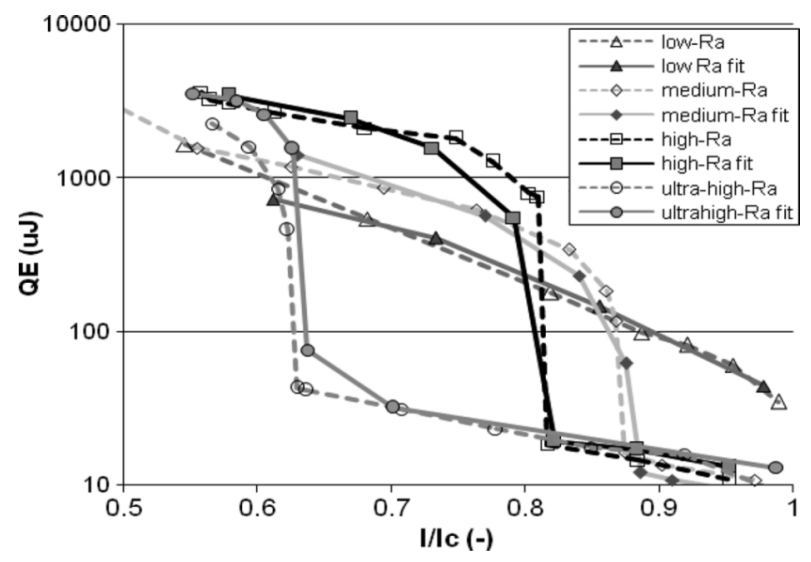

Fig. 8. Measured (dashed curves with markers) and calculated (solid lines) Quench Energy versus $I / I_{\mathrm{c}}$.

TABLE Y

OBTAINED FITTING PARAMETERS

\begin{tabular}{lll}
\hline \hline Identification & $a_{\text {trans }}\left[\mathrm{W} / \mathrm{m}^{2} / \mathrm{K}^{\mathrm{n}}\right]$ & $f_{\text {is }}\left[\mathrm{W} / \mathrm{m}^{2} / \mathrm{K}^{\mathrm{n}}\right]$ \\
\hline low- $R_{\mathrm{a}}$ & 50 & 1530 \\
medium- $R_{\mathrm{a}}$ & 140 & 50 \\
high- $R_{\mathrm{a}}$ & 77 & 30 \\
ultrahigh- $R_{\mathrm{a}}$ & 30 & 25 \\
\hline \hline
\end{tabular}

tance is highly dependent on the surface condition like oxidation and coating [16]. Both oxidization and solder coatings decrease the Kapitza conductance on which the transient conductance is based. This confirms the observed variation in $a_{\text {trans }}$. The untreated medium- $R_{\mathrm{a}}$ sample shows the highest value for $a_{\text {trans }}$.
Measurements by [17] on strands with the same coating show a comparable value for $a_{\text {trans }}$.

\section{CONCLUSIONS}

By applying four different kinds of treatments to a cored Rutherford cable we have varied $R_{\mathrm{a}}$, but at the same time also other parameters like RRR, $k_{\text {is }}$ and $k_{\mathrm{He}}$. Our stability measurements show that the Quench Energy versus $I / I_{\mathrm{c}}$ curves change drastically. The position of the so-called 'kink' in these curves is highly determining the cable stability and, as expected, the position of the kink is shifted to higher $I / I_{\mathrm{c}}$ with lower $R_{\mathrm{a}}$. The quench level left from the kink becomes higher for higher $R_{\mathrm{a}}$, mainly due to the decrease in $f_{\text {is }}$.

Depending on the $I / I_{\mathrm{C}}$ operating point our advice for the cable design for the SIS-300 magnets is to aim for the lowest possible $R_{\mathrm{a}}$ at which the AC loss is sufficiently low. The measurements show that an $R_{\mathrm{a}}$ of $650 \mu \Omega$ still gives a kink at $I / I_{\mathrm{c}}=$ 0.75 , a value which is sufficiently larger than the foreseen operating point of $I / I_{\mathrm{c}}=0.58$.

The results of the network model CUDI are in good agreement with the measurement results. The obtained fitting parameters are consistent with the expected parameter change due to the surface treatments of the samples.

\section{REFERENCES}

[1] M. N. Wilson and R. Wolf, "Calculation of minimum quench energies in Rutherford cable," IEEE. Trans. Appl. Supercond., vol. 7, p. 950, 1997.

[2] J. Kaugerts et al., "Cable design for FAIR SIS 300," IEEE. Trans. Appl. Supercond., vol. 17, p. 1477, 2007.

[3] A. P. Verweij et al., "1.9 K test facility for the reception of the superconducting cables for LHC," IEEE. Trans. Appl. Supercond., vol. 9, p. $153,1999$.

[4] A. Verweij, "CUDI: A model for calculation of electrodynamic and thermal behavior of superconducting Rutherford cables," Cryogenics, vol. 46, no. 7-8, p. 619, 2006, Used version 0701.

[5] D. Richter et al., "DC measurement of electrical contacts between strands insuperconducting cables for the LHC main magnets," IEEE. Trans. Appl. Supercond., vol. 7, p. 786, 1997.

[6] G. Willering and A. Verweij, "Investigation of Local Stability Behaviour of Superconducting NbTi Rutherford Cables by Measurements and Simulations With Point-Like Heaters," CERN AT-MAS internal note 2006-04, EDMS 788864, 2006.

[7] G. Willering et al., "New FRESCA Sample Holder for Cable Stability Experiments," internal note CERN-AT/MAS 2005-11, EDMS 691690.

[8] K. Seo, M. Morita, S. Nakamura, T. Yamada, and Y. Jizo, "Minimum quench energy measurement for superconducting wires," IEEE. Trans. Magn., vol. 32, p. 3089, 1996.

[9] A. Kimura et al., "Stabilities of Rutherford cables with CuNi matrix and CuMn barrier," IEEE. Trans. Appl. Supercond., vol. 5, p. 385, 1995.

[10] A. Gosh, W. Sampson, and M. Wilson, "Minimum quench energies of Rutherford cables and single wires," IEEE. Trans. Appl. Supercond., vol. 7, p. 1051, 1997.

[11] G. Willering et al., "Modeling the Heat Flow From a Graphite Paste Heater Used for Cable Stability Measurements," CERN AT-MCS, internal note 2007.

[12] J. Depond et al., "Superconducting Cable Topology," CERN LHC-MMS internal note 97-04, 1997.

[13] S. Van Sciver, Helium Cryogenics. New York: Plenum, 1986.

[14] C. Schmidt, "Transient heat transfer to liquid helium and temperature measurement with a response time in the microsecond region," Appl. Phys. Lett., vol. 32, p. 827, 1978.

[15] Y. Lei, Y. Yu, Y. Dai, and H. Nan, "Measurements of interstrand thermal and electrical conductance in multistrand superconducting cables," IEEE. Trans. Appl. Supercond., vol. 12, p. 1052, 2002.

[16] A. Kashani et al., "High heat flux Kapitza conductance of technical copper with several different surface preparations," Cryogenics, vol. 25 , no. 5 , p. $238,1985$.

[17] P. Bauer, "Stability of superconducting strands for accelerator magnets," Ph.D. dissertation, Technische Universitát, Wien, Austria, 2000. 